TITLE:

\title{
Two-dimensional vibrational spectroscopy of a double minimum system in a dissipative environment
}

$\operatorname{AUTHOR}(\mathrm{S}):$

Kuhn, O; Tanimura, Y

\section{CITATION:}

Kuhn, O ... [et al]. Two-dimensional vibrational spectroscopy of a double minimum system in a dissipative environment. JOURNAL OF CHEMICAL PHYSICS 2003, 119(4): 2155-2164

\section{ISSUE DATE:}

2003-07-22

URL:

http://hdl.handle.net/2433/49971

\section{RIGHT:}

Copyright 2003 American Institute of Physics. This article may be downloaded for personal use only. Any other use requires prior permission of the author and the American Institute of Physics. 


\title{
Two-dimensional vibrational spectroscopy of a double minimum system in a dissipative environment
}

\author{
O. Kühn ${ }^{\text {a) }}$ and Y. Tanimura ${ }^{\text {b) }}$ \\ Institute for Molecular Science, Nishigonaka 38, Myodaiji, Okazaki, Aichi 444-8585, Japan
}

(Received 10 March 2003; accepted 23 April 2003)

\begin{abstract}
A dissipative bistable system presents the simplest model to describe condensed phase reaction dynamics. Using a quantum master equation approach to calculate multitime dipole correlation functions we demonstrate how the dissipative dynamics can be characterized by time-resolved third-order infrared spectroscopy. Thereby we incorporate bilinear and linear-quadratic systembath interaction into the Redfield relaxation tensor. Investigating equilibrium and nonequilibrium initial conditions for a symmetric system it is shown that bath-induced coherence transfer can have a dramatic influence on the two-dimensional signals. This occurs when the inverse of the ground state tunneling splitting is of the order of the coherence transfer time. (c) 2003 American Institute of Physics. [DOI: 10.1063/1.1582841]
\end{abstract}

\section{INTRODUCTION}

Multidimensional vibrational spectroscopy ${ }^{1-3}$ has been proven to be a versatile tool to study such diverse topics as the inhomogeneity of liquids molecules, ${ }^{4}$ the anharmonicity of potentials, ${ }^{5-7}$ vibrational mode coupling, ${ }^{8-13}$ dephasing mechanisms, ${ }^{14-16}$ and structural changes of large molecules. ${ }^{17-22}$ For the fifth-order Raman spectroscopy, ${ }^{4}$ signals corresponding to various Raman polarizability tensor elements were measured for the intermolecular vibrational modes of liquid $\mathrm{CS}_{2}$ (Refs. 23-25) and solutions of $\mathrm{CS}_{2}$ (Ref. 26) by minimizing the cascade contributions, ${ }^{27,28}$ which were underestimated in the initial experiments. ${ }^{29-31}$ Theoretical analysis of the experimental results have been based on instantaneous normal modes ${ }^{32,33}$ and classical molecular dynamics simulations. ${ }^{34-36}$ For the third-order IR experiments, the two-dimensional (2D) Fourier plots of the three-pulse vibrational echo technique were applied to the conformational fluctuation of an $\alpha$-helical peptide, ${ }^{37}$ metal carbonyls, ${ }^{38}$ and model dipeptides. ${ }^{39}$ Theoretical studies of IR signals by means of molecular dynamics ${ }^{41,40}$ or quantum chemistry calculations ${ }^{42,43}$ were also carried out.

In this paper, we explore the possibility to scrutinize certain aspects of condensed phase molecular reaction $d y$ namics by means of multidimensional vibrational spectroscopy. Specifically, we will focus on isomerization reactions as characterized by a symmetric double well potential coupled to a heat bath. Having applications such as proton transfer reactions in mind, tunneling processes are of vital importance for understanding the reaction dynamics. The effect of tunneling can be seen in chemical reaction rates, ${ }^{44}$ in microwave, ${ }^{45}$ IR, ${ }^{46,47}$ or electronic absorption spectra. ${ }^{48}$

From the theoretical point of view a reaction can be described in terms of nuclear wave packet dynamics. ${ }^{49}$ In

a) Permanent address: Institut für Chemie, Physikalische und Theoretische Chemie, Freie Universtiät Berlin, Takustr. 3, 14195 Berlin, Germany; Electronic mail: ok@chemie.fu-berlin.de

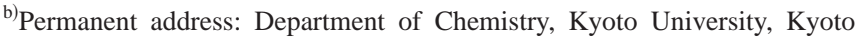
606-8502, Japan; Electronic mail: tanimura@kuchem.kyoto-u.ac.jp principle, the motion of a wave packet in a double well potential carries all the information. Unfortunately, the wave packet itself is not a physical observable and the profile of it is not so sensitive for a specific relaxation mechanism, a topic which plays a central role in the condensed phase. The chemical reaction rate, on the other hand, is a physical observable, but the change of the rate, e.g. with temperature, does not easily reveal microscopic details of the underlying mechanism. In this respect linear IR absorption or Raman spectra may provide more information, such as the frequency of the tunneling splitting levels. However, in the condensed phase, transition rates between different levels usually cannot unambiguously be extracted.

Both reaction rates and absorption spectrum are characterized by two-body (or two-time) correlation functions of physical operators such as the probability density ${ }^{50,51}$ and the dipole operators. ${ }^{52}$ For any physical operator $A(q)$, where $q$ is the molecular coordinate, the two-body correlation function is expressed as $\langle[A(q(t)), A(q(0))]\rangle$, where $\langle\ldots\rangle$ represents the ensemble average of the total system. The signals in multidimensional spectroscopy are characterized by multibody (multitime) correlation functions, e.g., $\left\langle\left[\left[A\left(q\left(t^{\prime}\right)\right), A(q(t))\right], A(q(0))\right]\right\rangle$. Studies of multidimensional spectroscopy have demonstrated that a profile of the multibody correlation function is very sensitive to details of the Hamiltonian (see, e.g., Ref. 14) as well as to the initial conditions. ${ }^{53}$ This implies that simulating multidimensional signals provides a very stringent test for the validity of a theoretical model. The reason can be found in the fact that the multibody correlation function is particularly sensitive to the anharmonicity of molecular motion. This can be seen by expanding the physical operator as $A(q)=c_{1} q+c_{2} q^{2}$. For a harmonic system, the leading order contribution in the twobody case is $c_{1}^{2}\langle[q(t), q(0)]\rangle$, whereas in the three-body case it is $c_{1}^{2} c_{2}\left\langle\left[\left[q\left(t^{\prime}\right), q(t)\right], q^{2}(0)\right]\right\rangle$, due to the Gaussian integral involved in the ensemble average. However, for an anharmonic system the leading order term in the three-body case is $c_{1}^{3}\left\langle\left[\left[q\left(t^{\prime}\right), q(t)\right], q(0)\right]\right\rangle$. Here, sources of anharmonicity may include, e.g., the quadratic-linear coordinate de- 
pendence of the mode-mode or the system-bath coupling.

We can discuss the same issue in the energy level representation, which is commonly used to study optical response functions. Here, the physical operators are expressed by the creation and annihilation operators, $a_{j}^{\dagger}$ and $a_{j}$, respectively, for the $j$ th energy level. This notation is especially useful in a frequency domain analysis, since the combination of the creation and annihilation operators such as $\left\langle\left[a_{j} a_{j+1}^{\dagger}, a_{j+1} a_{j}^{\dagger}\right]\right\rangle$ corresponds to combinations of level transitions at distinct frequencies. Similar to the coordinate representation, the leading-order contribution arises only when some sort of anharmonicity, which triggers dephasing or transitions between more than two different energy levels, plays a role. Since multidimensional spectroscopy uses more than two laser excitations, one can create many coherences to investigate the mechanism of level transitions caused by the relaxation processes or the anharmonicity. Tunneling processes in the energy level representation are characterized by pairs of states separated by the tunneling splitting. Since this gives rise to specific IR transitions multidimensional vibrational spectroscopy shall provide valuable information on the tunneling dynamics.

Traditionally, tunneling processes involved in chemical reactions are studied in the coordinate representation ${ }^{44} \mathrm{em}-$ ploying, for example, the path integral ${ }^{54}$ or the quantum Fokker-Planck equation approach. ${ }^{55}$ Although path integral calculations of multitime correlation functions have been performed, e.g., for a two-state system, ${ }^{56}$ the optical response accompanying tunneling processes in multilevel systems is more conveniently investigated by means of an appropriate quantum master equation for the reduced density operator in the energy level representation. ${ }^{57-60}$ Regardless of the representation, the different approaches should describe the same dynamics. However, in the case of optical response one usually employs additional nontrivial approximations such as the rotating wave approximation, i.e., the various approaches are no longer equivalent. While such approximations might be fully justified for electronic transitions, they do not necessarily give an adequate description for IR transitions. As it was shown that multidimensional spectroscopy is very sensitive to the details of the response functions, ${ }^{16}$ it can be expected to provide a unique tool for studying the validity of the underlying equations of motion.

In this paper, we will address this issue by simulating multitime correlation functions using a quantum master equation approach where energy and phase relaxation is described in terms of the so-called Redfield tensor. ${ }^{57-60}$ Thereby we include bilinear and quadratic-linear systembath coupling for the Hamiltonian in the coordinate representation. This gives rise to one- and two-quantum relaxation and dephasing, but also to pure dephasing; these processes are usually described by phenomenological times scales $T_{1}$, $T_{2}$, and $T_{2}^{*}$, respectively. ${ }^{14}$ This corresponds to the Bloch limit of the Redfield tensor. Recently, Tannor and Kohen have given an illuminating discussion of the various approximations to the Redfield tensor in terms of the phase space dynamics of a harmonic oscillator. ${ }^{61}$ In the present contribution we will demonstrate that for the anharmonic double minimum potential, the Bloch limit and full Redfield dynam-

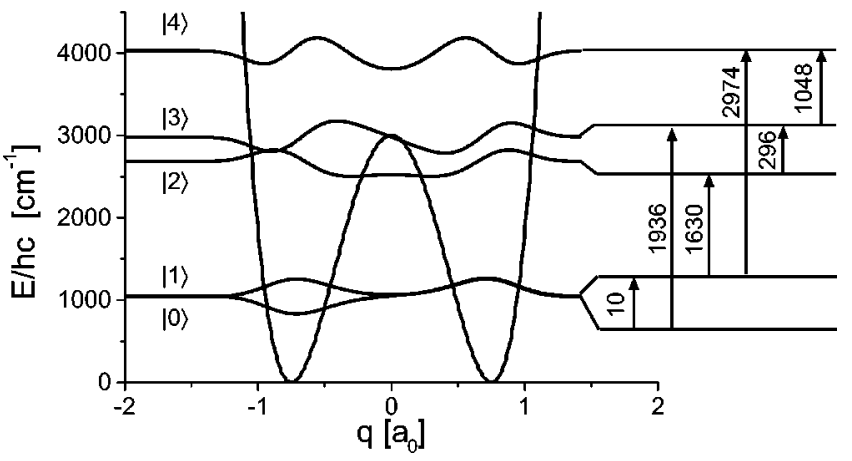

FIG. 1. Potential Eq. (1) with the lowest eigenstate wave functions at the respective eigenenergies for $q_{0}=0.77 a_{0}$ and $V_{B} / h c=3000 \mathrm{~cm}^{-1}$. The dipole-allowed IR transitions according to Eq. (15) are shown as well (in $\left.\mathrm{cm}^{-1}\right)$. The coordinate matrix elements are $\langle 0|q| 1\rangle=0.71,\langle 0|q| 3\rangle=0.16$, $\langle 1|q| 2\rangle=0.18,\langle 1|q| 4\rangle=0.06,\langle 2|q| 3\rangle=0.5,\langle 3|q| 4\rangle=0.40$ (in $a_{0}$. .) Equation (7) has been diagonalized using the Fourier Grid Hamiltonian method (Ref. 77) using 1024 grid points in the interval $\left[-4 a_{0}: 4 a_{0}\right]$. In the numerical solution of Eq. (6) the eight lowest states have been taken into account $\left(\omega_{70} / 2 \pi c=6406 \mathrm{~cm}^{-1}\right)$.

ics can lead to significantly different signals in twodimensional vibrational spectroscopy.

The paper is organized as follows: In Sec. II we will introduce the model for the system-bath Hamiltonian. Section III summarizes the calculation of nonlinear response functions. Numerical results for equilibrium and nonequilibrium initial conditions are presented in Sec. IV. Conclusions are summarized in Sec. V.

\section{MODEL SYSTEM}

We consider a one-dimensional system described by a reaction coordinate $q$ and the following symmetric bistable potential:

$$
V(q)=\frac{V_{B}}{q_{0}^{4}}\left(q-q_{0}\right)^{2}\left(q+q_{0}\right)^{2},
$$

where $V_{B}$ is the height of the barrier separating the two minima which are located at $\pm q_{0}$ as shown in Fig. 1. All other degrees of freedom of the total system, i.e., intramolecular and solvent modes, will be comprised in a (effective) harmonic bath with the system-bath interaction Hamiltonian being of the form ${ }^{58}$

$$
H_{\mathrm{SB}}=\sum_{u} K^{(u)}(q) \Phi^{(u)}\left(\left\{Z_{\xi}\right\}\right) .
$$

Here the operators $K^{(u)}$ and $\Phi^{(u)}$ belonging to the system and the bath, respectively. In principle one can write down explicit expressions for $H_{\mathrm{SB}}$ starting from a Taylor expansion of the global potential energy surface. For proton transfer reactions, for instance, one can expect that intramolecular modes modulating the proton transfer distance will give the dominant contribution. The leading terms in Eq. (2) are of linear and quadratic order with respect to the proton transfer coordinate. Note that the quadratic coupling is usually considered to be of great importance for it leads to a promotion of the proton transfer by effectively reducing the reaction barrier. ${ }^{58}$ A two-dimensional dissipative model explicitly including a promoting mode has been studied. ${ }^{62}$ However, in 
order to keep the present model as simple as possible such that the information content of 2D IR spectroscopy can be clearly identified, we will use a one-dimensional description. This is reasonable provided that the coupling to the bath is weak and the coupling strength is distributed over many modes. $^{63}$

In the following we will take into account the bilinear $(L)$ and the quadratic-linear $(Q)$ coupling, i.e., the sum in Eq. (2) contains two terms, $u=(L, Q)$, with

$$
\begin{aligned}
& K^{(L)}(q)=q / q_{0}, \\
& K^{(Q)}(q)=\left(q / q_{0}\right)^{2},
\end{aligned}
$$

and

$$
\Phi^{(L / Q)}\left(\left\{Z_{\xi}\right\}\right)=\sum_{\xi} \hbar \Omega_{\xi} g_{L / Q}(\xi) \widetilde{Z}_{\xi}
$$

Here $\Omega_{\xi}$ and $g_{u}(\xi)$ are the frequency and dimensionless coupling strength, respectively, for the $\xi$ th bath mode. Further, we used dimensionless bath coordinates $\widetilde{Z}_{\xi}$ $=Z_{\xi}\left(2 M_{\xi} \Omega_{\xi} / \hbar\right)^{1 / 2}$.

Within second-order perturbation theory with respect to $H_{\mathrm{SB}}$ and using the Markov approximation the equation of motion for the reduced statistical operator of the system, i.e., $\rho=\operatorname{tr}_{B} \rho_{\text {tot }}$, can be written as ${ }^{58}$

$$
\frac{\partial \rho}{\partial t}=-i \hat{L}_{\mathrm{S}} \rho-\hat{R} \rho
$$

with the Liouville superoperator for the system being $\hat{L}_{\mathrm{S}} \bullet$ $=\left[H_{\mathrm{S}}, \cdot\right] / \hbar$. The effect of the system-bath interaction is contained in the (Redfield) relaxation superoperator $\hat{R}$. The matrix elements of $\hat{R}$ in the basis of the eigenstates of the system Hamiltonian, i.e.,

$$
H_{\mathrm{S}}|a\rangle=\left[\frac{p^{2}}{2 m}+V(q)\right]|a\rangle=E_{a}|a\rangle
$$

can be expressed in terms of the damping matrix

$$
\Gamma_{a b, c d}(\omega)=\sum_{u u^{\prime}} K_{a b}^{(u)} K_{c d}^{\left(u^{\prime}\right)} C_{u u^{\prime}}(\omega)
$$

as $^{58-60}$

$$
\begin{aligned}
R_{a b, c d}= & \delta_{a c} \sum_{e} \Gamma_{b e, e d}\left(\omega_{d e}\right)+\delta_{b d} \sum_{e} \Gamma_{a e, e c}\left(\omega_{c e}\right) \\
& -\Gamma_{c a, b d}\left(\omega_{d b}\right)-\Gamma_{d b, a c}\left(\omega_{c a}\right) .
\end{aligned}
$$

Here, $K_{a b}^{(u)}=\left\langle a\left|K^{(u)}(q)\right| b\right\rangle$ is the matrix element of Eq. (3) or (4) for the energy eigenstates $|a\rangle$ and $|b\rangle$. In Eq. (8) the Fourier transform of the bath equilibrium correlation function has been introduced as [the imaginary part of the damping matrix is neglected; $n(\omega)$ is the Bose-Einstein distribution function]

$$
C_{u u^{\prime}}(\omega)=\pi(1+n(\omega))\left(J_{u u^{\prime}}(\omega)-J_{u u^{\prime}}(-\omega)\right),
$$

which depends on the spectral density

$$
\begin{aligned}
J_{u u^{\prime}}(\omega) & =\omega^{2} \sum_{\xi} g_{u}(\xi) g_{u^{\prime}}(\xi) \delta\left(\omega-\Omega_{\xi}\right) \\
& \approx g_{u} g_{u^{\prime}} \Theta(\omega) \omega \exp \left\{-\omega / \omega_{c}\right\} .
\end{aligned}
$$

To keep the matter simple we have assumed that there is a single spectral density of Ohmic form with cut-off frequency $\omega_{c}$ characterizing the linear and quadratic coupling.

It is well known that quadratic coupling leads to pure dephasing. In terms of the Redfield tensor this implies that the damping function with frequency argument zero becomes important. To disentangle the effect of pure dephasing from that of two-quantum transitions we will characterize pure dephasing by the parameter $\gamma_{\mathrm{pd}}$ having the dimensions of $\mathrm{s}^{-1}$. To this end we write ${ }^{64,65}$

$$
\lim _{\omega \rightarrow 0} C_{u u^{\prime}}(\omega)=g_{u} g_{u^{\prime}} \gamma_{\mathrm{pd}}
$$

To summarize, the system-bath interaction is characterized by four parameters, i.e., $g_{L}, g_{Q}, \gamma_{\mathrm{pd}}$, and $\omega_{c}$.

The multilevel Redfield equations are often discussed within two limiting cases, the simplest one being the Bloch limit which migrated from the field of nuclear magnetic resonance spectroscopy into vibrational relaxation theory. It assumes that populations and coherences within the density matrix are completely decoupled such that

$$
\sum_{c d} R_{a b, c d} \rho_{c d} \rightarrow\left(1-\delta_{a b}\right) R_{a b, a b} \rho_{a b}+\delta_{a b} \sum_{c} R_{a a, c c} \rho_{c c} .
$$

Here $R_{a b, a b}$ and $R_{a a, c c}$ find a straightforward interpretation as coherence dephasing and population relaxation rates, respectively, which in the limit of a two-level system lead to the well-known $T_{2}$ and $T_{1}$ times. The validity of the Bloch limit can be appreciated by looking at the secular approximation to the equations of motion. Here, in the spirit of a rotating wave approximation, one retains only those terms on the right-hand-side which obey $\left|\omega_{a b}-\omega_{c^{\prime} d^{\prime}}\right|=0$, i.e.,

$$
\sum_{c d} R_{a b, c d} \rho_{c d} \rightarrow \sum_{c^{\prime} d^{\prime}} R_{a b, c^{\prime} d^{\prime}} \rho_{c^{\prime} d^{\prime}}
$$

Apparently, the contributions kept in the Bloch limit survive the secular approximation. In addition, however, the righthand side of Eq. (14) mixes different coherence matrix elements, i.e., a bath-induced coherence transfer (CT) $\rho_{a b}$ $\rightarrow \rho_{c^{\prime} d^{\prime}}$ occurs. ${ }^{66-69}$ Beyond the secular approximation the bath-induced conversion of coherences into populations, $\rho_{a b} \rightarrow \rho_{c c}$, and vice versa becomes possible as well. ${ }^{70,71}$

The primary example for the importance of non-Bloch terms leading to $\mathrm{CT}$ within the secular approximation is the dissipative harmonic oscillator. ${ }^{61}$ Here the bilinear coupling gives nonzero matrix elements of $K_{a b}^{(L)}$ for $a=b \pm 1 \quad(a$ $>b$ ), i.e., CT like $\rho_{a b} \rightarrow \rho_{a \pm 1, b \pm 1}$ becomes possible. For the quadratic-linear coupling $K_{a b}^{(Q)} \neq 0$ holds for $a=b \pm 2$ ( $a$ $>b+1)$ and for $a=b$. Consequently, CT proceeds like $\rho_{a b}$ $\rightarrow \rho_{a \pm 2, b \pm 2}$. If both coupling mechanisms are simultaneously present, Eq. (8) also contains cross-terms such as $\propto K_{a a \pm 1}^{(L)} K_{a a \pm 2}^{(Q)}$. Strictly speaking these contributions do not survive the secular approximation. However, for a sufficiently strong system-bath coupling such that $R_{a a \pm 1, a a \pm 2}$ 
$\approx \omega_{10}$ CT might still be possible. Indeed it has been shown by using a quantum Fokker-Planck equation approach that such cross-terms can have a large impact on multidimensional spectroscopic signals. ${ }^{16}$

The situation in the present double minimum system is different in several respects. First, we notice that as a consequence of the symmetry we have $K_{a b}^{(L)} \neq 0$ for $a=2 i,(2 i$ $+1)$ and $b=2 j+1,(2 j)$ and $K_{a b}^{(Q)} \neq 0$ for $a=2 i,(2 i+1)$ and $b=2 j,(2 j+1)$ with $i, j=0,1, \ldots$. Thus in principle the number of possible transitions is much larger than in the harmonic oscillator case. Second, in the case that bilinear and quadratic-linear system-bath coupling are considered separately, the conditions for the secular approximation are hardly fulfilled due to the anharmonicity of the potential. Third, and most importantly, the interplay between crossterms in the interaction and the energy level structure can lead to an efficient CT even for a weak system-bath coupling. Inspecting Fig. 1 we notice that this CT should involve the tunneling doublets by virtue of processes like $\rho_{2 i, 2 j}$ $\rightarrow \rho_{2 i+1,2 j}(i<j=0,1, \ldots)$. Following the above-given reasoning this requires that $R_{2 i, 2 j, 2 i+1,2 j} \approx \omega_{2 i+1,2 i}$. In particular the ground state tunneling splitting can become rather small, e.g., for high barrier systems, such that even the original condition of the secular approximation will be almost fulfilled. At first sight it may appear as if this limit is of no relevance in the context of ultrafast reaction dynamics. However, in Sec. IV we will demonstrate that CT involving the ground state doublet can have a dramatic influence on the spectroscopy of higher excited tunneling pairs where the tunneling dynamics takes place on a much faster time scale.

\section{IR RESPONSE FUNCTION}

The response of the molecular system to an external field $\mathbf{E}(\mathbf{r}, t)$ is conveniently described in terms of multitime correlation functions. We will assume the matter-field interaction Hamiltonian to be of the form

$$
H_{F}(t)=-\mu_{0} q E(t) \text {. }
$$

Thus only the one-dimensional system interacts via its dipole operator $\mu(q)=\mu_{0} q$ with the field which is treated within the long-wavelength limit. In third order with respect to $E(t)$ the polarization is given by ${ }^{52}$

$$
\begin{aligned}
P^{(3)}(t)= & \int_{0}^{\infty} d t_{3} d t_{2} d t_{1} R^{(3)}\left(t_{3}, t_{2}, t_{1}\right) E\left(t-t_{3}\right) \\
& \times E\left(t-t_{3}-t_{2}\right) E\left(t-t_{3}-t_{2}-t_{1}\right)
\end{aligned}
$$

with the response function being defined as a function of the three time intervals between the interactions

$$
\begin{aligned}
R^{(3)}\left(t_{3}, t_{2}, t_{1}\right)= & \left(\frac{i}{\hbar}\right)^{3} \mu_{0}^{4} \operatorname{tr}\left\{q \hat { G } ( t _ { 3 } ) \left[q, \hat{G}\left(t_{2}\right)\left[q, \hat{G}\left(t_{1}\right)\right.\right.\right. \\
& \left.\left.\left.\times\left[q, \rho_{0}\right]\right]\right]\right\} .
\end{aligned}
$$

Here $\hat{G}(t)$ is the Liouville space Green's function and $\rho_{0}$ is the initial state density operator, normally being equal to the equilibrium statistical operator. $R^{(3)}\left(t_{3}, t_{2}, t_{1}\right)$ can be calculated analytically, e.g., for the harmonic Brownian oscillator model. ${ }^{4,5,11}$ Here we aim at a numerical simulation for the system introduced in Sec. II. To simplify matters we will assume that the trace over the bath degrees of freedom can be performed for each time interval separately. ${ }^{52}$ This implies a phase randomization between the different propagation intervals which allows us to write

$$
\hat{G}(t) \rightarrow \hat{G}_{\mathrm{S}}(t)=\Theta(t) \exp \left\{-i \hat{L}_{\mathrm{S}} t-\hat{R} t\right\} .
$$

In the following we will be interested in the information contained in $R^{(3)}\left(t_{3}, t_{2}, t_{1}\right)$ itself, i.e., we assume the impulsive limit where the signal is proportional to the response function. For finite pulse envelopes, the signal depends not only on the pulse form but also on the experimental layout due to the phase-matching condition. ${ }^{72}$ To characterize the resonances in $R^{(3)}\left(t_{3}, t_{2}=0, t_{1}\right)$ we will calculate the frequency domain signal according to

$$
S\left(\Omega_{3}, \Omega_{1}\right)=\int_{0}^{\infty} d t_{3} d t_{1} e^{i \Omega_{3} t_{3}+i \Omega_{1} t_{1}} R^{(3)}\left(t_{3}, t_{2}=0, t_{1}\right) .
$$

The population dynamics will be investigated by means of the frequency-dispersed homodyne detected signal which is defined as

$$
S_{\text {hom }}\left(\Omega_{3}, t_{2}, t_{1}\right)=\left|\int_{0}^{\infty} d t_{3} e^{i \Omega_{3} t_{3}} R^{(3)}\left(t_{3}, t_{2}, t_{1}\right)\right|^{2} .
$$

\section{NUMERICAL RESULTS}

In this section we will pursue two goals, i.e., to characterize the dissipative dynamics of the double minimum potential by means of the third-order IR response function and, at the same time, to compare Bloch limit (BL) and full Redfield (FR) theory. In order to validate a rotating wave approximation, Eq. (14), $\left|\omega_{a b}-\omega_{c d}\right|^{-1}$ for those terms which are neglected should be much larger than a typical time scale for the dissipative evolution of the density matrix. ${ }^{58}$ As has been discussed in Sec. II this condition might not be fulfilled in dissipative tunneling systems where the tunneling splittings can be of the order of a few tens of $\mathrm{cm}^{-1}$. The parameters of the present simulation have been chosen such as to highlight this issue. As an example we will consider the case $q_{0}=0.77 a_{0}$ and $V_{B} / h c=3000 \mathrm{~cm}^{-1}$ shown in Fig. 1 . If the particle is assumed to have the mass of a proton these parameters give rise to two tunneling doublets, i.e., for the ground state with $\omega_{10} / 2 \pi c=10 \mathrm{~cm}^{-1}$ and for an excited state with $\omega_{32} / 2 \pi c=296 \mathrm{~cm}^{-1}$.

\section{A. Equilibrium initial conditions}

Let us consider the nonlinear IR response of the symmetric potential of Fig. 1 starting from a canonical equilibrium density matrix at the temperature $T=300 \mathrm{~K}$. In Fig. 2 the response function $R^{(3)}\left(t_{3}, t_{2}=0, t_{1}\right)$ is shown for the linear system-bath coupling case as obtained within the BL (a) and from the FR equations (b). The most striking observation is that while in the $\mathrm{BL} R^{(3)}$ is decaying within a few hundred femtoseconds, in the FR case it does not decay within the 2 ps for which it is plotted along the $t_{3}$ direction.

This unexpected behavior can be shown to be due to bath induced CT which comes into play because the transition frequency associated with the tunneling doublet is rather 

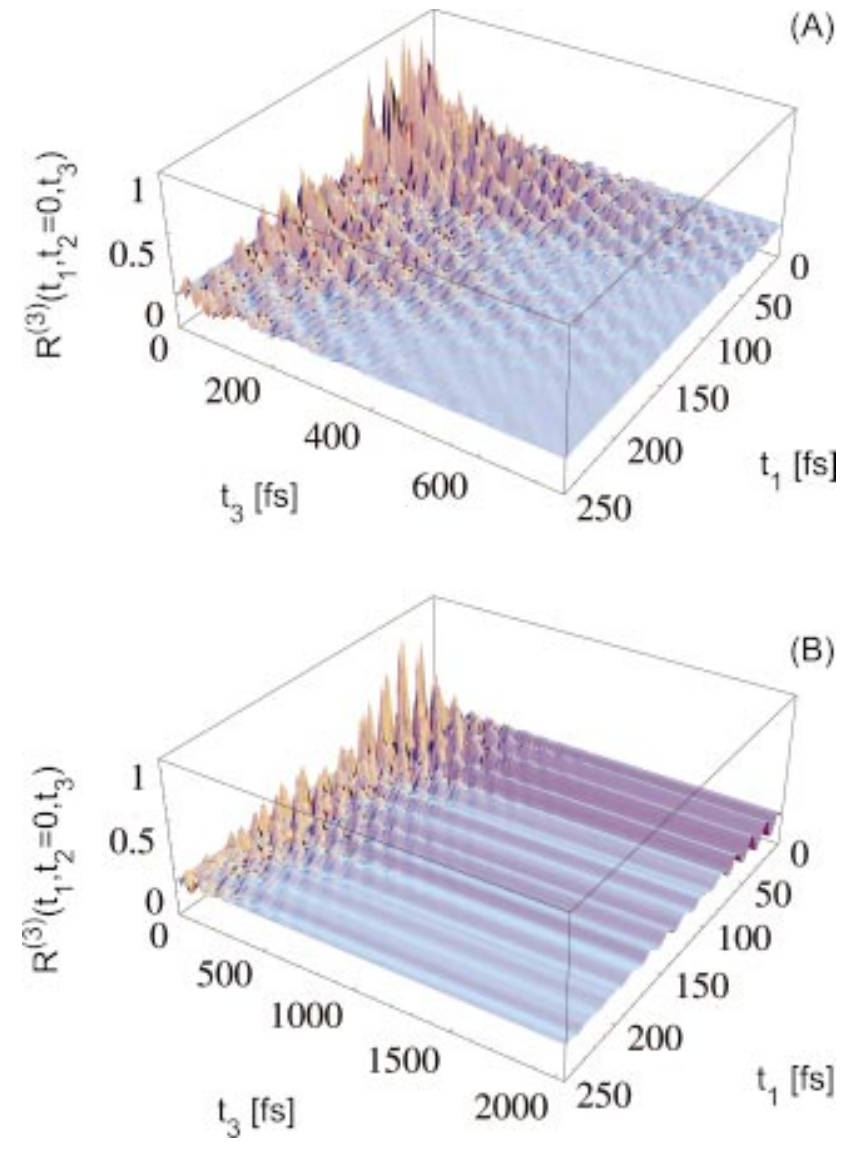

FIG. 2. (Color) Third-order response function $R^{(3)}\left(t_{3}, 0, t_{1}\right)$ (in arbitrary units) as obtained for the system in Fig. 1 using the BL (a) and the FR tensor (b). The system-bath coupling parameters are: $g_{L}=0.5, g_{Q}=0, \gamma_{\mathrm{pd}}=0$, and $\omega_{c} / 2 \pi c=500 \mathrm{~cm}^{-1}(T=300 \mathrm{~K})$. Notice that in the FR case the response functions becomes constant along both time axes.

small, i.e., $\left|\omega_{10}-\omega_{01}\right| / 2 \pi c=20 \mathrm{~cm}^{-1}$, and the related time scale is long compared to that of the density matrix evolution. To see this let us consider the density matrix evolution for the ground state tunneling doublet only. Taking into account CT terms and contributions with positive frequency arguments in the damping matrix only one obtains

$$
\begin{aligned}
\frac{\partial}{\partial t} \rho_{10} & =-i \omega_{10} \rho_{10}-R_{10,10} \rho_{10}-R_{10,01} \rho_{01} \\
& =-i \omega_{10} \rho_{10}-2 i \Gamma_{10,01}\left(\omega_{10}\right) \operatorname{Im} \rho_{10} .
\end{aligned}
$$

Here we made use of the fact that $R_{10,10}=-R_{10,01}$ $\approx \Gamma_{10,01}\left(\omega_{10}\right)$. In the BL, on the other hand, one has

$$
\frac{\partial}{\partial t} \rho_{10}=-i \omega_{10} \rho_{10}-\Gamma_{10,01}\left(\omega_{10}\right) \rho_{10} .
$$

For a given initial condition both limits give comparable results as long as the damping is small, i.e., the real and imaginary parts of $\rho_{10}$ simultaneously decay to zero. If the coupling strength increases such that the associated dephasing rate $\Gamma_{10,01}$ exceeds $\omega_{10}$ the BL just gives some acceleration of the decay while the solution of the FR equations is qualitatively different. That is, because only $\operatorname{Im} \rho_{10}$ appears on the right-hand side of Eq. (21), the real and imaginary parts of $\rho_{10}$ are treated rather differently which leads to an overall deceleration of the decay with increasing coupling strength. Solving Eq. (21) analytically for $\Gamma_{10,01} \gg \omega_{10}$ even gives $\rho_{10}(t) \sim \operatorname{Re} \rho_{10}(0)+i \operatorname{Im} \rho_{10}(0) \exp \left\{-2 \Gamma_{10,01} t\right\}$. For the parameters used in Fig. 2 we have $R_{10,10} / 2 \pi c=70 \mathrm{~cm}^{-1}$, i.e., this limit is realized and the response function becomes a constant after some initial decay. Of course, this behavior merely demonstrates the breakdown of the second-order perturbation theory. However, to make the point this breakdown is only observed when including coherence transfer into the equations of motion; the BL will give reasonable looking results even beyond the limits of its applicability.

Being aware of these problems we can turn to the real effect of bath-induced CT and nonsecular relaxation dynamics. What comes to mind immediately is that CT might lead to the appearance of resonances which are otherwise not observable. Provided that both the system-bath coupling and the dipole operator depend linearly on the reaction coordinate only resonances at the dipole allowed transitions will be observable, most notably at $\Omega_{1 / 3}=\omega_{30} / \omega_{21}$. If we allow for a quadratic dependence of the system-bath coupling on the reaction coordinate, bath-induced $\mathrm{CT}$ in principle should give new resonances by virtue of processes like $\rho_{2 i, 2 j}$ $\rightarrow \rho_{2 i, 2 j+1}(i<j=0,1,2, \ldots)$ as discussed in Sec. II. However, they will not be directly observable due to the linearity of the dipole operator.

In Fig. 3 we compare $\left|S\left(\Omega_{3}, \Omega_{1}\right)\right|$ as obtained from the BL [(a) and (b)] and the FR equations [(c) and (d)] including linear and quadratic system-bath coupling, but neglecting pure dephasing, i.e., the damping matrices $\Gamma_{a a, b b}(0)$ are zero. Let us consider the region of the fundamental transitions $0 \rightarrow 3\left(\omega_{30} / 2 \pi c=1936 \mathrm{~cm}^{-1}\right)$ and $1 \rightarrow 2\left(\omega_{21} / 2 \pi c\right.$ $\left.=1630 \mathrm{~cm}^{-1}\right)\left[(\mathrm{a})\right.$ and (c)]. A typical Feynman diagram ${ }^{52}$ contributing to the diagonal peaks is shown in Fig. 4(a). The system-bath coupling parameters have been chosen such that both transitions have a comparable dephasing rate in the $\operatorname{BL}\left(R_{30,30} / 2 \pi c=19 \mathrm{~cm}^{-1}\right.$ and $\left.R_{21,21} / 2 \pi c=20 \mathrm{~cm}^{-1}\right)$. The most notable difference to the FR signal is that for the latter the widths of the resonances are considerably reduced as seen from Fig. 3(c). This is due to the coupled dynamics of coherences, in the present case according to the CT mechanisms

$$
\begin{gathered}
\rho_{21} \stackrel{R_{21,20}}{\longrightarrow} \rho_{20} \stackrel{R_{20,21}}{\longrightarrow} \rho_{21}, \\
\rho_{30} \stackrel{R_{30,31}}{\longrightarrow} \rho_{31} \stackrel{R_{31,30}}{\longrightarrow} \rho_{30} .
\end{gathered}
$$

The respective Redfield tensor elements are $R_{21,20} / 2 \pi c$ $=-16 \mathrm{~cm}^{-1}$ and $R_{30,31} / 2 \pi c=-5 \mathrm{~cm}^{-1}$. Close inspection of Eqs. (8) and (9) shows that the dominant contributions are $R_{21,20} \propto g_{L} g_{Q} q_{10}\left(q_{11}^{2}-q_{22}^{2}\right)$ and $R_{30,31} \propto g_{L} g_{Q} q_{10}\left(q_{00}^{2}-q_{33}^{2}\right)$, where $q_{a b}=\langle a|q| b\rangle$ (see the following). For the symmetric potential in Fig. 1 we have $q_{00}^{2} \sim q_{11}^{2}$ but $q_{22}^{2}<q_{33}^{2}$, since the odd number state within the first excited tunneling doublet has its node at $q=0$. Therefore, the effect of bath-induced $\mathrm{CT}$ is more pronounced for the lower-frequency transition $1 \rightarrow 2$. This modification giving an effective damping is indeed strong enough to significantly change the relative amplitudes of the considered resonances. While all peaks shown are of similar amplitude in the BL, there is a clear preference 
(A)

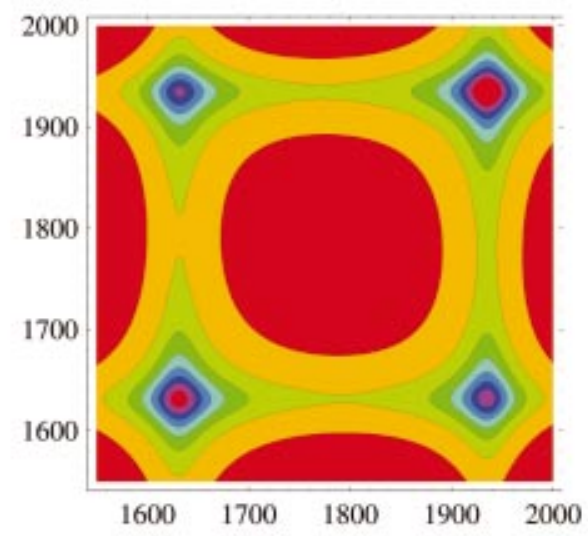

(B)

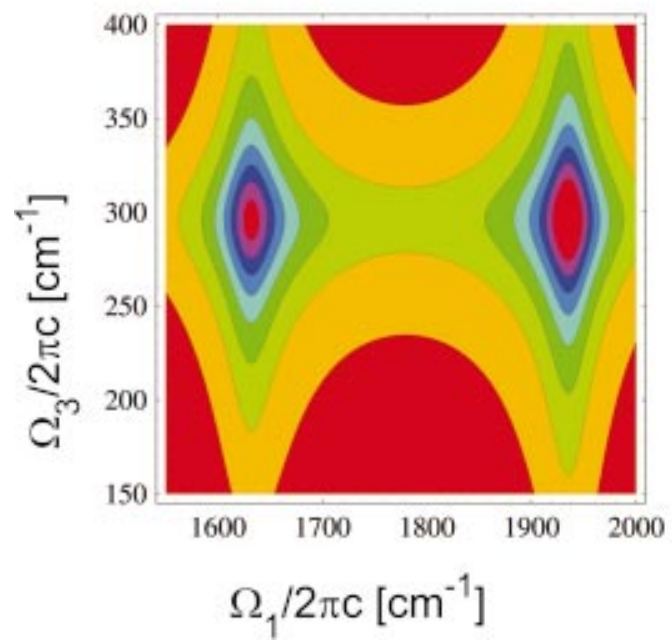

(C)

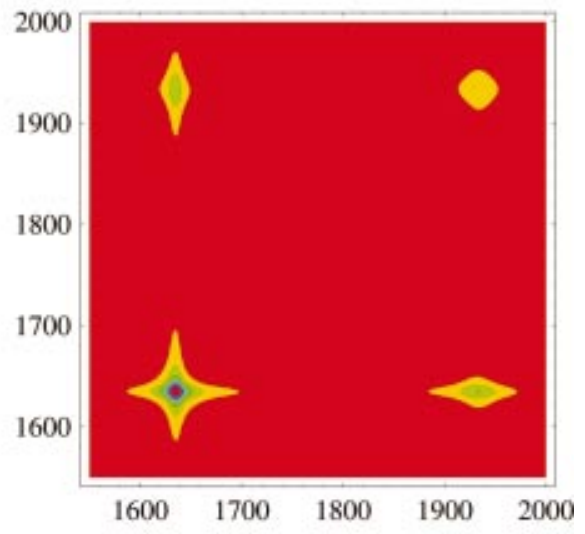

(D)

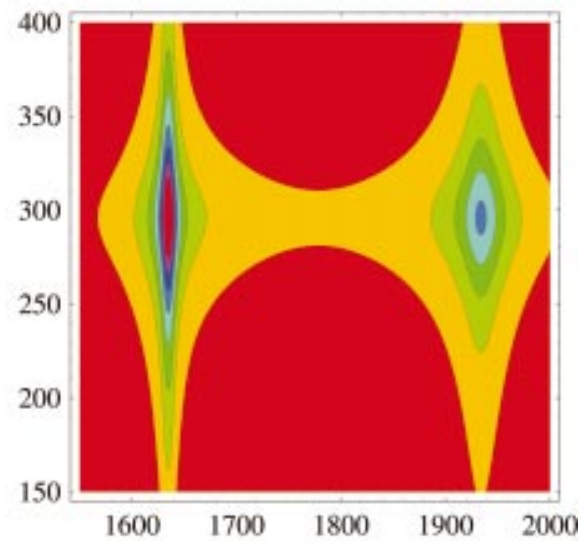

FIG. 3. (Color) Frequency domain signal $\left|S\left(\Omega_{3}, \Omega_{1}\right)\right|$ for the BL [(a) and (b)] and using the FR tensor [(c) and (d)]. The relevant transition frequencies are: $\omega_{21}=1630, \omega_{30}=1936$, and $\omega_{32}=296 \mathrm{~cm}^{-1}$. The system-bath parameters are: $g_{L}=0.19, g_{Q}=3.0, \gamma_{\mathrm{pd}}=0$, and $\omega_{c} / 2 \pi c=500 \mathrm{~cm}^{-1}$. The normalization of each panel is such that the amplitude for the strongest peak at about $\left(\omega_{30}, \omega_{30}\right)(a),\left(\omega_{30}, \omega_{32}\right)(b),\left(\omega_{21}, \omega_{21}\right)(\mathrm{c}),\left(\omega_{21}, \omega_{32}\right)(d)$ is unity; the contours are drawn at $0.1,0.2, \ldots, 0.8$.

for the $\left(\Omega_{1}, \Omega_{3}\right) \approx\left(\omega_{21}, \omega_{21}\right)$ resonance in the FR case. Simultaneously, the off-diagonal peaks at $\left(\Omega_{1}, \Omega_{3}\right)$ $\sim\left(\omega_{21}, \omega_{30}\right)$ and $\left(\omega_{30}, \omega_{21}\right)$ take an elongated shape along the $\Omega_{3}$ and $\Omega_{1}$ axes, respectively.

Next we have a closer look at the positions of the resonances. As anticipated there is no observable double-peak structure of the individual peaks due to the tunneling splitting. Considering the diagonal peaks only, we notice that there is, however, a shift in the peak position into the direction of the IR forbidden transitions $0 \rightarrow 2 \quad\left(\omega_{20} / 2 \pi c\right.$ $\left.=1640 \mathrm{~cm}^{-1}\right)$ and $1 \rightarrow 3\left(\omega_{31} / 2 \pi c=1926 \mathrm{~cm}^{-1}\right)$. The diagonal peaks are observed at $(1934,1934) \mathrm{cm}^{-1}$ and $(1635,1635) \mathrm{cm}^{-1}$, i.e., there is a shift of -2 and $+5 \mathrm{~cm}^{-1}$ for the $0 \rightarrow 3$ and $1 \rightarrow 2$ transitions, respectively. This is a manifestation of bath-induced CT contributions.

To understand the two effects of non-Bloch type relaxation, i.e., change of the width and position of resonances, in more detail, let us consider a three level system comprised of the states $|0\rangle,|1\rangle,|2\rangle$ of the present model. Looking only at the coherences and taking into account CT induced by $R_{20,21}$ one can write down two coupled equations of motion for $\rho_{21}$ and $\rho_{20}$. Solving for $\rho_{20}$, inserting the result into the equation for $\rho_{21}$, and invoking a Markov-type approximation one obtains an equation of motion for $\rho_{21}$ which contains an effective frequency and damping:

$$
\begin{aligned}
& \omega_{21}^{(\mathrm{eff})}=\omega_{21}+\left(\omega_{20}-\omega_{21}\right) \frac{R_{21,20} R_{20,21}}{\left(\omega_{20}-\omega_{21}\right)^{2}+R_{20,20}^{2}}, \\
& R_{21,21}^{\text {(eff) }}=R_{21,21}-R_{20,20} \frac{R_{21,20} R_{20,21}}{\left(\omega_{20}-\omega_{21}\right)^{2}+R_{20,20}^{2}} .
\end{aligned}
$$

This result clearly shows that the non-Bloch terms lead to a decrease of the transition frequency as well as of the damping for the $1 \rightarrow 2$ transition. Note that for the $0 \rightarrow 3$ the frequency shift is negative since $\omega_{30}>\omega_{31}$.

In Fig. 3 we also compare the BL (b) with the solution of the FR equations (d) for the case that in the second coherence period, i.e., during $t_{3}$, a low-frequency transition is excited. In the present case the respective transition is 2 $\rightarrow 3\left(\omega_{32} / 2 \pi c=296 \mathrm{~cm}^{-1}\right)$. Again we notice that the FR dynamics is rather different from the BL. The fact that CT 
(A)

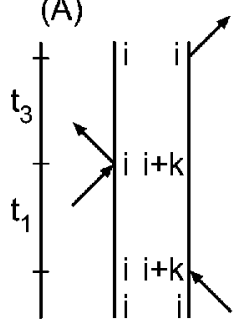

(B)

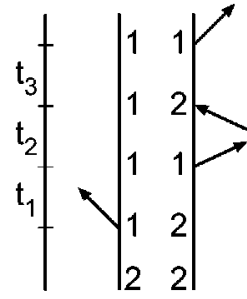

(C)

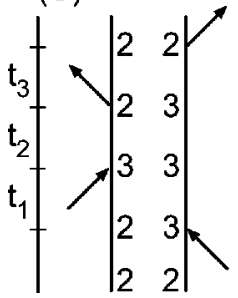

(D)

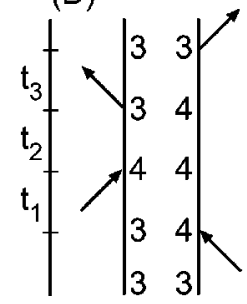

FIG. 4. Double sided Feynman diagrams contributing to the third-order response function for the symmetric double minimum system in Fig. 1. (a) Diagram which is influenced by the CT mechanism leading to the "dressing" of transition energies and dephasing rates as given in Eqs. (23) and (24). For the example in Fig. 3[(a) and (c)] $(i=0, k=3)$ and $(i=1, k=1)$ holds for the diagonal peaks at $\left(\omega_{30}, \omega_{30}\right)$ and $\left(\omega_{21}, \omega_{21}\right)$, respectively. (b)(d) Typical diagrams relevant for the response after initial preparation in the superposition state $(|2\rangle+|3\rangle) / \sqrt{2}$ as shown in Fig. 6. Diagrams like (b)-(d) are discussed as cases (i)-(iii) in Sec. IV B.

has a stronger influence on the $1 \rightarrow 2$ transition is reflected in the narrowing of the respective (left) peak. This causes a considerable change in the amplitudes shifting the maximum in panel (d) to $\left(\Omega_{1}, \Omega_{3}\right) \approx\left(\omega_{21}, \omega_{32}\right)$ while the two peaks were of similar amplitude in the BL (b). Closer inspection shows that there is a small shift of the resonance position along the $\Omega_{1}$ axis due to CT processes related to $\omega_{31}$ and $\omega_{20}$. However, the $2 \rightarrow 3$ transition is rather unaffected by non-Bloch terms in the relaxation matrix. In fact, neither the width nor the position along the $\Omega_{3}$ axis are appreciably modified. This is not surprising since all possible transitions for CT are far off-resonant.

In Fig. 5 we compare the BL (a) with the FR dynamics (b) for a case where pure dephasing is included. The parameters have been chosen such that the dephasing rate for the $1 \rightarrow 2$ transition does not change as compared to Fig. 3. This amounts to changing the ratio $\gamma_{\mathrm{pd}} / g_{Q}$ and thus the relative importance of zero- and two-quantum transitions. As a consequence of the symmetry and its particularly pronounced effect on the matrix elements of $q^{2}$ the dephasing rates and therefore the relative magnitudes of the $1 \rightarrow 2$ and $0 \rightarrow 3$ transitions become different. This is reflected in $S\left(\Omega_{3}, \Omega_{1}\right)$ already in the BL in Fig. 5(a). Specifically, one obtains for the pure dephasing contribution $R_{a b, a b}^{(\mathrm{pd})}=\gamma_{\mathrm{pd}} g_{Q}^{2}\left(q_{a a}^{2}-q_{b b}^{2}\right)^{2}$. Keeping only the dominant terms from the population relaxation related dephasing one has

$$
\begin{gathered}
R_{21,21} \sim\left|q_{10}\right|^{2} C_{L L}\left(\omega_{10}\right)+\left|q_{20}^{2}\right|^{2} C_{Q Q}\left(\omega_{20}\right) \\
+\gamma_{\mathrm{pd}} g_{Q}^{2}\left(q_{22}^{2}-q_{11}^{2}\right)^{2}, \\
R_{30,30} \sim\left|q_{10}\right|^{2} C_{L L}\left(\omega_{01}\right)+\left|q_{31}^{2}\right|^{2} C_{Q Q}\left(\omega_{31}\right) \\
+\gamma_{\mathrm{pd}} g_{Q}^{2}\left(q_{33}^{2}-q_{00}^{2}\right)^{2} .
\end{gathered}
$$

(A)
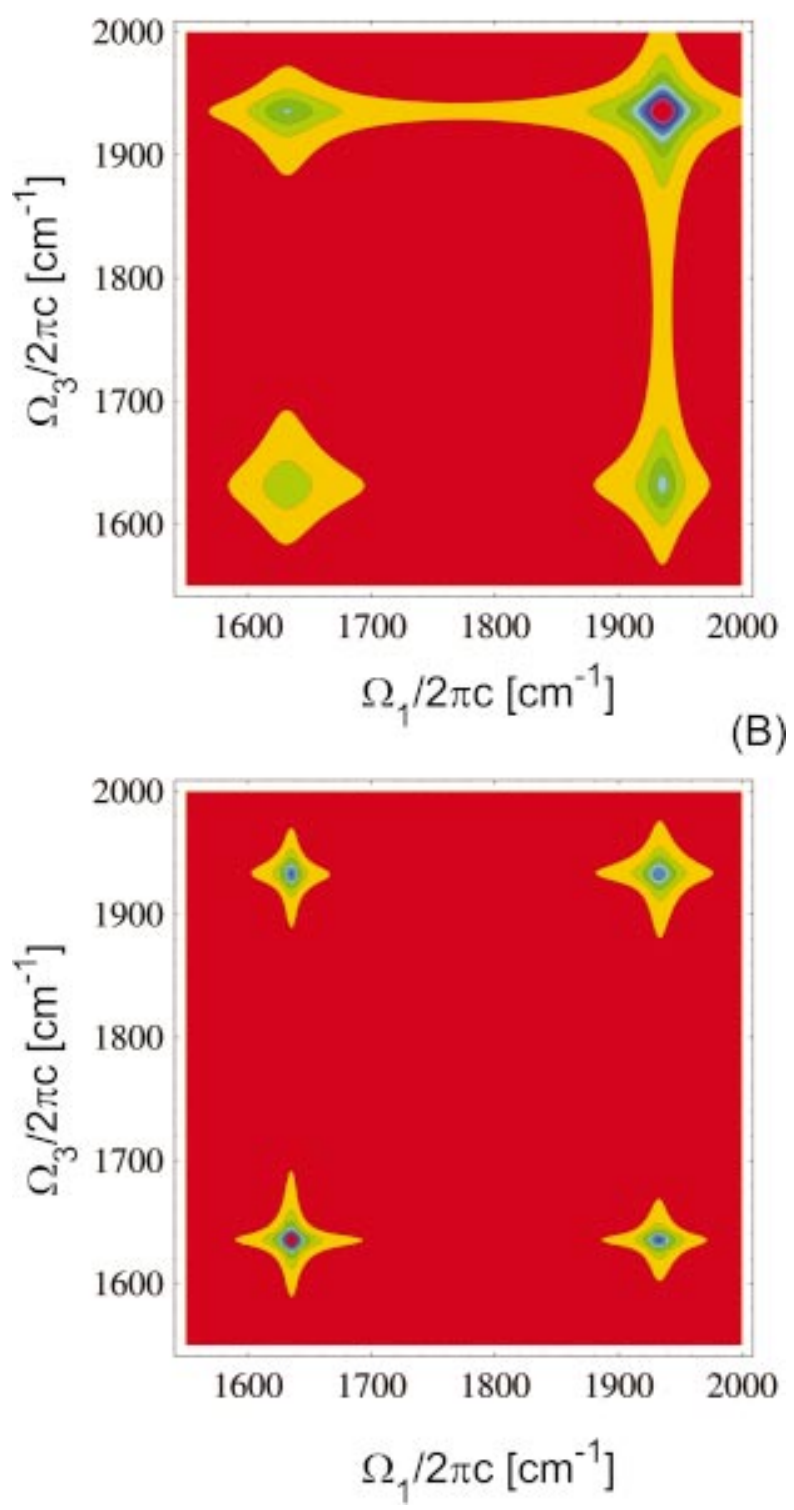

FIG. 5. (Color) Same as Figs. 3(a) and 3(c) but with inclusion of pure dephasing; BL—panel (a), FR—panel (b). The system-bath parameters are: $g_{L}=0.19, g_{Q}=1.1, \gamma_{\mathrm{pd}}=0.005$, and $\omega_{c} / 2 \pi c=500 \mathrm{~cm}^{-1}$.

In the present simulation the first term is kept constant while the relative contribution from the second and third terms is varied. Apparently, if $R_{a b, a b}$ is dominated by pure dephasing as in Fig. 5(a) the observed change in relative amplitudes is due to $R_{21,21}^{\mathrm{pd}} / R_{30,30}^{\mathrm{pd}}>1$. In passing we note that for the same reason the influence of pure dephasing on $R_{10,10}$ is negligible since $q_{00}^{2} \sim q_{11}^{2}$.

Upon inclusion of all Redfield tensor elements the same effect as in the case with no pure dephasing (cf. Fig. 3) is seen. However, here it approximately compensates for the redistribution of amplitudes introduced by pure dephasing. This is a consequence of the dependence of the CT Redfield tensor elements on the ratio $\gamma_{\mathrm{pd}} / g_{Q}$ which is different from that of the elements responsible for coherence dephasing. Noting that $R_{a b, a d}^{(\mathrm{pd})}=\gamma_{\mathrm{pd}} g_{L} g_{Q} q_{b d}\left(q_{d d}^{2}-q_{a a}^{2}\right)$ and keeping 

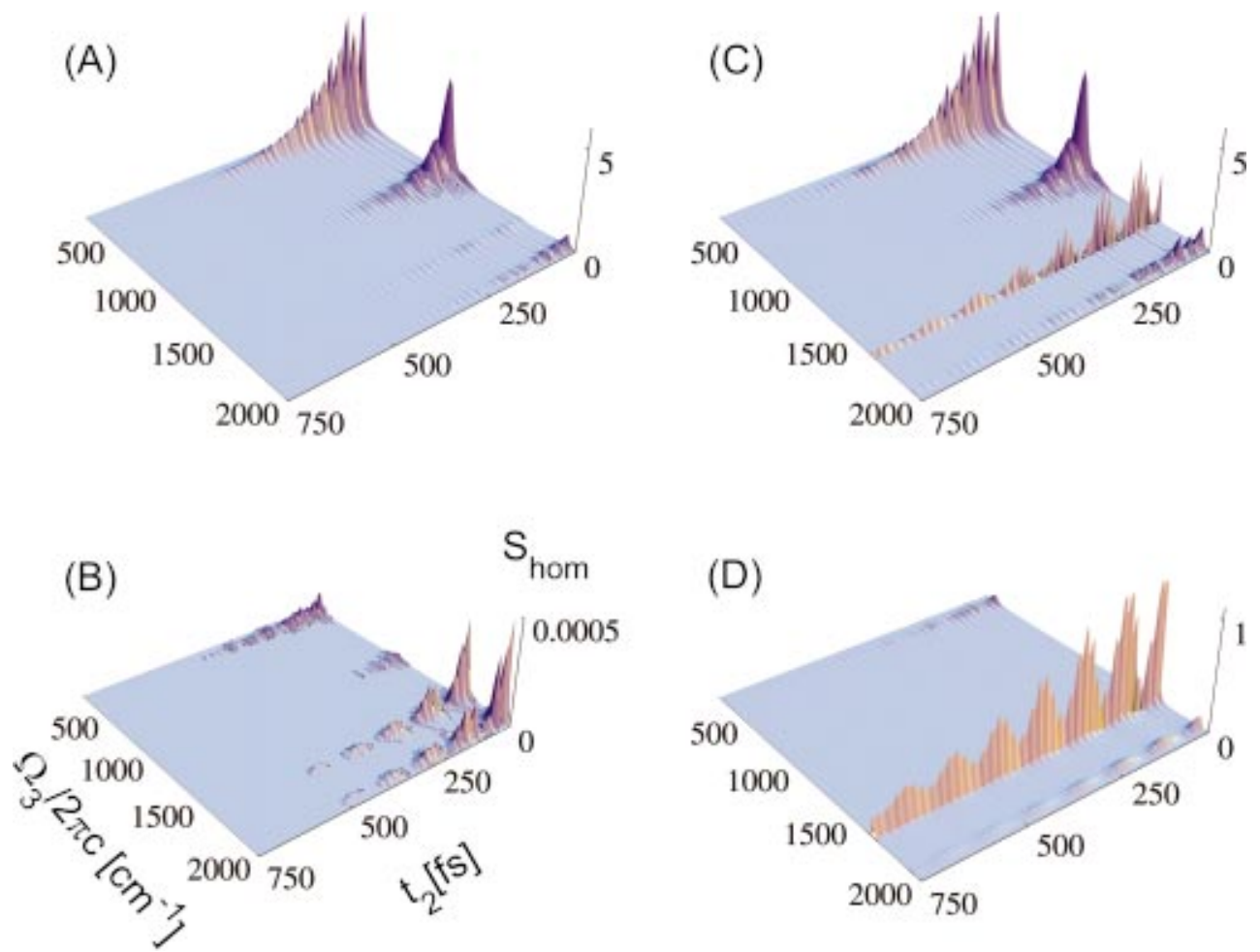

FIG. 6. (Color) Homodyne signal Eq. (20) for a system initially prepared in the nonequilibrium state $(|2\rangle+|3\rangle) / \sqrt{2}$. The BL $[(a)$ and (b)] and the FR dynamics $\left[(\mathrm{c})\right.$ and (d)] is shown for $t_{1}=0\left[(\mathrm{a})\right.$ and (c)] and $t_{1}=1000 \mathrm{fs}\left[(\mathrm{b})\right.$ and (d)]. The relevant transitions frequencies are: $\omega_{32}=296, \omega_{43}=1048, \omega_{21}=1630$, and $\omega_{30}=1936 \mathrm{~cm}^{-1}$. The system-bath parameters are: $g_{L}=0.19, g_{Q}=3, \gamma_{\mathrm{pd}}=0$, and $\omega_{c} / 2 \pi c=500 \mathrm{~cm}^{-1}$. Notice that the rather strong resonance at $\Omega_{3}=\omega_{10}$ is not shown for clarity.

only dominant terms from the remaining contributions to Eq. (9) one obtains

$$
\begin{aligned}
& R_{21,20} \sim\left|q_{10}\right|\left(q_{00}^{2}-q_{22}^{2}\right)\left[C_{L Q}\left(\omega_{01}\right)+g_{L} g_{Q} \gamma_{\mathrm{pd}}\right], \\
& R_{30,31} \sim\left|q_{10}\right|\left(q_{11}^{2}-q_{33}^{2}\right)\left[C_{L Q}\left(\omega_{10}\right)+g_{L} g_{Q} \gamma_{\mathrm{pd}}\right] .
\end{aligned}
$$

Thus changing $\gamma_{\mathrm{pd}} / g_{Q}$ will merely modify the relative contributions of the two terms to the square brackets. The $\left(q_{d d}^{2}\right.$ $-q_{a a}^{2}$ ) dependence responsible for the different behavior of the $1 \rightarrow 2$ and $0 \rightarrow 3$ transitions is not influenced. This result of course reflects the fact that mixed linear-quadratic interactions are responsible for bath-induced CT. Coherence dephasing, on the other hand, is due to linear-linear and quadratic-quadratic interactions.

\section{B. Nonequilibrium initial conditions}

In the following we will discuss the IR response function including population relaxation during the $t_{2}$ period. As it was shown previously, ${ }^{53}$ multidimensional spectroscopy has the capability to measure not only the position but also the momentum of a wave packet. Since a chemical reaction process is a movement of the wave packet and the dynamics starting from an equilibrium state are rather unspectacular, we focus on the time evolution of a nonequilibrium state. Specifically, we assume that the system has been prepared in the superposition state $(|2\rangle+|3\rangle) / \sqrt{2}$ which is localized to the right of the barrier. We envisage such a state, e.g., as to emerge from a laser control scheme or to be part of the initial state nuclear wave packet after some photoexcitation process.
In Fig. 6 we show the homodyne signal, $S_{\text {hom }}\left(\Omega_{3}, t_{2}, t_{1}\right)$, in the spectral range also covered in Fig. 3 for the BL without $\left[t_{1}=0\right.$, panel (a) $]$ and with $\left[t_{1}\right.$ $=1000 \mathrm{fs}$, panel (b)] evolution during the first coherence period. We can distinguish three types of resonances which evolve during the $t_{2}$ period in (i) one of the states of the ground state tunneling doublet, (ii) one of the states of the excited state tunneling doublet, and (iii) some higher excited (delocalized) above barrier state. Typical Feynman diagrams are shown in Figs. 4(b)-4(d). In Fig. 6 these resonances give rise to peaks at $\Omega_{3}=\omega_{30}$ and $\omega_{21}$ (i), $\Omega_{3}=\omega_{32}$ (ii), and $\Omega_{3}$ $=\omega_{43}$ (iii). (Notice that due to the linearity of the dipole operator only the diagonal elements of the initial density matrix contribute to the signal.) As a consequence of the larger transition dipole matrix elements, $S_{\text {hom }}\left(\Omega_{3}, t_{2}, t_{1}=0\right)$ shown in Fig. 6(a) is dominated by type (ii) and (iii) resonances. Generally speaking the higher the quantum number the faster will be the population relaxation for a considered state. Consequently, the type (iii) resonance at $\Omega_{3}=\omega_{43}$ decays more rapidly along the $t_{2}$ axis as compared to the type (ii) resonance at $\Omega_{3}=\omega_{32}$. Since the dephasing rates increase as well for the higher excited states, allowing coherence evolution during $t_{1}$ leads to the disappearance of the peaks in the order type (iii), (ii), and finally (i). Therefore, in panel (b) the signal is dominated by the type (i) resonances at $\Omega_{3}=\omega_{30}$ and $\omega_{21}$.

The situation changes for the FR case as can be seen from the corresponding panels (c) and (d) of of Fig. 6. As discussed in the previous section, the resonance for the 1 $\rightarrow 2$ transition will be considerably narrowed due to CT. In 
Fig. 6(c) this compensates for the smaller transition dipole matrix element and-in contrast to Fig. 6(a) - the type (i) resonance at $\Omega_{3}=\omega_{21}$ is clearly observed. Inspection of this resonance shows that the relaxation during the $t_{2}$ period is slowed down as well. Again the type (ii) and (iii) resonances are the first to disappear if coherence evolution during $t_{1}$ is included [Fig. 6(d)].

As a consequence of the long decay time of the $\Omega_{3}$ $=\omega_{21}$ resonance a slow modulation of the signal along the $t_{2}$ axis can be clearly recognized. This feature, also present in the $\Omega_{3}=\omega_{30}$ resonance and in the BL, is due to the damped wave packet oscillation between left and right potential wells; the oscillation period being determined by the splitting of the excited state tunneling doublet. Viewed from another perspective, this is a manifestation of the isomerization reaction dynamics in the two-dimensional IR signal.

\section{CONCLUSIONS}

In recent years multidimensional spectroscopy has emerged as a powerful tool for investigating structural and dynamical correlations in molecular systems. While previous theoretical work had been focused on nonreactive situations, the present contribution is to our knowledge the first study of a condensed phase reactive multilevel system. Our emphasis was put on the dynamics of a reaction coordinate moving in a symmetric double minimum potential under the influence of a bilinear and a quadratic-linear system-bath coupling. For the sake of clarity the system parameters were chosen such that there were two pairs of states below the reaction barrier giving rise to symmetry-allowed transitions which could be easily distinguished. The situation is exemplary, e.g., for proton transfer across weak $\mathrm{H}$ bonds.

Recent ultrafast experiments on H-bonded systems provided evidence for subpicosecond phase and energy relaxation time scales. ${ }^{73-75}$ The information about the time scales is usually extracted from the experimental data using either a Brownian oscillator description-in close analogy to the treatment of electronic transitions-or a simple phenomenological Bloch-type $T_{1}$ and $T_{2}$ parametrization. As far as the proton transfer reaction envisaged in the present work is concerned it is reasonable to assume that the phase and energy relaxation time scales are likely to be much shorter than the time scale for tunneling within the ground state doublet. Viewed from the perspective of a microscopic modeling, this constellation is throwing some doubts on the applicability of the Bloch model.

This issue has been addressed here by comparing twodimensional IR signals from the full Redfield equations with those obtained within the Bloch limit. The differences have been demonstrated to be striking; the most notable effects being a reduction of the linewidths, the modification of relative amplitudes of resonance peaks, and the slowing down of population relaxation. They can be traced back to the bathinduced coherence transfer which becomes possible due to transitions which are resonant within the width of the small ground state tunneling splitting.

We would like to emphasize that this coherence transfer mechanism has an influence on the observation of the tunneling reaction dynamics of vibrationally excited states which usually will be much faster than the time scale of ground state tunneling. Further, it is important to note that for the present symmetric system such coherence transfer is caused by the combined effect of bilinear and quadraticlinear system-bath coupling. For the treatment of nonreactive system the latter is normally not included, although it can lead to a distinct multidimensional signal as shown recently. ${ }^{14}$ For the reactive case as encountered in proton transfer systems, however, the quadratic interaction with bath modes in general cannot be neglected. Here such modes are known as gating modes for they can promote the proton transfer by reducing the reaction barrier. ${ }^{58}$

The present results have been obtained under the assumption of a weak system-bath coupling in the white noise limit. The most obvious improvement would start from an enlarged system, i.e., by including a strongly coupled mode explicitly into the relevant part which is then treated using Redfield theory. ${ }^{62,69,76}$ On the other hand, the quantum Fokker-Planck equation approach has been shown to be suitable to incorporate Gaussian-Markovian heat baths. ${ }^{14,16,55}$ Being formulated in phase space, however, the computational effort for including more than one degree of freedom into the relevant system becomes prohibitive. Still another alternative is given by a path integral formulation for the equilibrium correlation functions. ${ }^{56}$ Here one is restricted to a few eigenstates of the relevant system. The success of this approach therefore depends on the possibility to map the system-bath interaction onto a spectral density which gives rise to short correlation times only. However, it is important to emphasize that relaxation processes induced by a quadratic-linear system-bath coupling in the coordinate representation of the Hamiltonian are usually not taken into account in the path integral approach. No matter what the specific approach is, multidimensional spectroscopy will provide a stringent test of its validity when it comes to the comparison with experimental data.

\section{ACKNOWLEDGMENTS}

This work has been financially supported by the a Grantin-Aid for Scientific Research (B) No. (12440171) from Japan Society for the Promotion of Science (Y.T.) and Morino Science Foundation (Y.T.), and the Fonds der Chemischen Industrie (O.K.). O.K. is grateful for the hospitality of the Institute for Molecular Science in Okazaki.

${ }^{1}$ S. Mukamel, Annu. Rev. Phys. Chem. 51, 691 (2000).

${ }^{2}$ J. T. Fourkas, Adv. Chem. Phys. 117, 235 (2001).

${ }^{3}$ J. C. Wright, Int. Rev. Phys. Chem. 21, 185 (2002).

${ }^{4}$ Y. Tanimura and S. Mukamel, J. Chem. Phys. 99, 9496 (1993).

5 (a) K. Okumura and Y. Tanimura, J. Chem. Phys. 106, 1687 (1997); (b) 107, 2267 (1997).

${ }^{6}$ Y. Tanimura, Chem. Phys. 233, 217 (1998).

${ }^{7}$ R. Akiyama and R. F. Loring, J. Chem. Phys. 116, 4655 (2002).

${ }^{8}$ A. Tokmakoff, M. J. Lang, D. S. Larsen, G. R. Fleming, V. Chernyak, and S. Mukamel, Phys. Rev. Lett. 79, 2702 (1997).

${ }^{9}$ K. Okumura and Y. Tanimura, Chem. Phys. Lett. 278, 175 (1997).

${ }^{10}$ M. Cho, K. Okumura, and Y. Tanimura, J. Chem. Phys. 108, 1326 (1998).

${ }^{11}$ K. Okumura, D. M. Jonas, and Y. Tanimura, Chem. Phys. 266, 237 (2001).

${ }^{12}$ N.-H. Ge, M. T. Zanni, and R. M. Hochstrasser, J. Phys. Chem. A 106, 962 (2002).

${ }^{13}$ R. Venkatramani and S. Mukamel, J. Chem. Phys. 117, 11089 (2002). 
${ }^{14}$ (a) T. Steffen and Y. Tanimura, J. Phys. Soc. Jpn. 69, 3115 (2000); (b) Y. Tanimura and T. Steffen, ibid. 69, 4095 (2000).

${ }^{15}$ Y. Tanimura, in Two-Dimensional Correlation Spectroscopy, edited by Y. Ozaki and I. Noda (Wiley, New York, 2002), p. 144.

${ }^{16}$ (a) T. Kato and Y. Tanimura, J. Chem. Phys. 6221, 117 (2002); (b) (unpublished).

${ }^{17}$ P. Hamm, M. Lim, and R. M. Hochstrasser, J. Phys. Chem. B 102, 6123 (1998).

${ }^{18}$ K. Okumura, A. Tokmakoff, and Y. Tanimura, J. Chem. Phys. 111, 492 (1999).

${ }^{19}$ W. Zhao and J. C. Wright, Phys. Rev. Lett. 84, 1411 (2000).

${ }^{20}$ M. C. Asplund, M. T. Zanni, and R. M. Hochstrasser, Proc. Natl. Acad. Sci. U.S.A. 97, 8219 (2000)

${ }^{21}$ (a) C. Scheurer, A. Piryatinski, and S. Mukamel, J. Am. Chem. Soc. 123, 3114 (2001); (b) C. Scheurer and S. Mukamel, J. Chem. Phys. 116, 6803 (2002).

${ }^{22}$ K. A. Merchant, W. G. Noid, D. E. Thompson, R. Akiyama, R. F. Loring, and M. D. Fayer, J. Phys. Chem. B 107, 4 (2003).

${ }^{23}$ K. J. Kubarych, C. L. Milne, S. Lin, V. Astinov, and J. D. Miller, J. Chem. Phys. 116, 2016 (2002).

${ }^{24}$ O. Golonzka, N. Demirdöven, M. Khalil, and A. Tokmakoff, J. Chem. Phys. 113, 9893 (2000).

${ }^{25}$ L. J. Kaufman, J. Heo, L. D. Ziegler, and G. R. Fleming, Phys. Rev. Lett. 88, 207402 (2002).

${ }^{26} \mathrm{~K}$. Tominaga and K. Yoshihara, J. Chin. Chem. Soc. (Taipei) 47, 631 (2000).

${ }^{27}$ J. C. Kirkwood and A. C. Albrecht, J. Raman Spectrosc. 31, 107 (2000).

${ }^{28}$ M. Cho, D. A. Blank, J. Sung, K. Park, S. Hahn, and G. R. Fleming, J. Chem. Phys. 112, 2082 (2000).

${ }^{29}$ K. Tominaga and K. Yoshihara, Phys. Rev. Lett. 74, 3061 (1995).

${ }^{30}$ T. Steffen and K. Duppen, Phys. Rev. Lett. 76, 1224 (1996).

${ }^{31}$ A. Tokmakoff and G. R. Fleming, J. Chem. Phys. 106, 2569 (1997).

${ }^{32}$ (a) T. Keyes and J. T. Fourkas, J. Chem. Phys. 112, 287 (2000); (b) R. L. Murry, J. T. Fourkas, and T. Keyes, ibid. 109, 7913 (1998).

${ }^{33}$ (a) A. Ma and R. M. Stratt, Phys. Rev. Lett. 85, 1004 (2000); (b) J. Chem. Phys. 116, 4972 (2002).

${ }^{34}$ (a) S. Saito and I. Ohmine, J. Chem. Phys. 108, 240 (1998); (b) 106, 4889 (1997).

${ }^{35}$ (a) T. I. C. Jansen, J. G. Snijders, and K. Duppen, J. Chem. Phys. 113, 307 (2000); (b) 114, 10910 (2001).

${ }^{36}$ S. Saito and I. Ohmine, Phys. Rev. Lett. 88, 207401 (2002).

${ }^{37}$ (a) S. Woutersen and P. Hamm, J. Phys. Chem. B 104, 11316 (2000); J. Chem. Phys. 115, 7737 (2001).

${ }^{38}$ (a) O. Golonzka, M. Kahlil, N. Demirdöven, and A. Tokmakoff, J. Chem. Phys. 115, 10814 (2001); (b) N. N. Demirdöven, M. Kahlil, O. Golonzka, and A. Tokmakoff, J. Phys. Chem. A 105, 8025 (2001); (c) N. Demirdöven, M. Khalil, and A. Tokmakoff, Phys. Rev. Lett. 89, 237401 (2002).

${ }^{39}$ (a) M. T. Zanni, S. Gnanakaran, J. Stenger, and R. M. Hochstrasser, J. Phys. Chem. B 105, 6520 (2001); (b) I. V. Rubtsov and R. M. Hochstrasser, ibid. 106, 9165 (2002).

${ }^{40}$ S. Gnanakaran and R. M. Hochstrasser, J. Am. Chem. Soc. 123, 12886 (2001).

${ }^{41}$ S. Woutersen, R. Pfister, P. Hamm, Y. Mu, D. S. Kosov, and G. Stock, J. Chem. Phys. 117, 6833 (2002).

${ }^{42}$ S. Cha, S. Ham, and M. Cho, J. Chem. Phys. 117, 740 (2002)

${ }^{43}$ (a) A. M. Moran, S. M. Park, J. Dreyer, and S. Mukamel, J. Chem. Phys.
118, 3651 (2003); (b) A. M. Moran, J. Dreyer, and S. Mukamel, ibid. 118, 1347 (2003).

${ }^{44}$ P. Hänggi, P. Talkner, and M. Borkovec, Rev. Mod. Phys. 62, 251 (1990), and references therein.

${ }^{45}$ K. Tanaka, H. Honjo, T. Tanaka, H. Kohguchi, Y. Ohshima, and Y. Endo, J. Chem. Phys. 110, 1969 (1999).

${ }^{46}$ R. L. Redington, T. E. Redington, and J. M. Montgomery, J. Chem. Phys. 113, 2304 (2000).

${ }^{47}$ F. Madeja and M. Havenith, J. Chem. Phys. 117, 7162 (2002).

${ }^{48}$ H. Sekiya, Y. Nagashima, and Y. Nishimura, J. Chem. Phys. 92, 5761 (1990).

${ }^{49}$ J. Manz, in Femtochemistry and Femtobiology, edited by V. Sundström (Imperial College Press, London, 1997), p. 80.

${ }^{50}$ T. Yamamoto, J. Chem. Phys. 33, 281 (1960).

${ }^{51}$ D. Chandler, J. Chem. Phys. 68, 2959 (1978).

${ }^{52}$ S. Mukamel, Principles of Nonlinear Optical Spectroscopy (Oxford University Press, New York, 1995).

${ }^{53}$ Y. Suzuki and Y. Tanimura, J. Chem. Phys. 115, 2267 (2001).

${ }^{54}$ A. J. Leggett, Phys. Rev. B 30, 1208 (1984).

${ }^{55}$ (a) Y. Tanimura and P. G. Wolynes, Phys. Rev. A 43, 4131 (1991); (b) J. Chem. Phys. 96, 8485 (1992).

${ }^{56}$ J. Shao and N. Makri, J. Chem. Phys. 116, 507 (2002).

${ }^{57}$ W. T. Pollard, A. K. Felts, and R. A. Friesner, Adv. Chem. Phys. 93, 77 (1996).

${ }^{58}$ V. May and O. Kühn, Charge and Energy Transfer Dynamics in Molecular Systems (Wiley-VCH, Berlin, 2000).

${ }^{59}$ A. G. Redfield, Adv. Magn. Reson. 1, 1 (1965).

${ }^{60}$ K. Blum, Density Matrix Theory and Application (Plenum, New York, 1981).

${ }^{61}$ D. Kohen and D. J. Tannor, Adv. Chem. Phys. 111, 219 (2000).

${ }^{62}$ (a) N. Došlić, K. Sundermann, L. González, O. Mó, J. Giraud-Girard, and O. Kühn, Phys. Chem. Chem. Phys. 1, 1249 (1999); (b) N. Došlić and O. Kühn, Chem. Phys. 255, 247 (2000).

${ }^{63}$ (a) H. Naundorf, K. Sundermann, and O. Kühn, Chem. Phys. 240, 163 (1999); (b) O. Kühn, Eur. Phys. J. D 6, 49 (1999); (c) O. Kühn, Y. Zhao, F. Shuang, and Y.-J. Yan, J. Chem. Phys. 112, 6104 (2000).

${ }^{64}$ D. Reichman, R. J. Silbey, and A. Suarez, J. Chem. Phys. 105, 10500 (1996).

${ }^{65}$ Y. Yan, F. Shuang, R. Xu, J. Cheng, X.-Q. Li, C. Yang, and H. Zhang, J. Chem. Phys. 113, 2068 (2000).

${ }^{66}$ Y. Ohtsuki and Y. Fujimura, J. Chem. Phys. 91, 3903 (1989).

${ }^{67}$ J. M. Jean and G. R. Fleming, J. Chem. Phys. 103, 2092 (1995).

${ }^{68}$ D. Egorova, A. Kuhl, and W. Domcke, Chem. Phys. 268, 105 (2001).

${ }^{69}$ O. Kühn and H. Naundorf, Phys. Chem. Chem. Phys. 5, 79 (2003).

${ }^{70}$ A. M. Walsh and R. D. Coalson, Chem. Phys. Lett. 198, 293 (1992).

${ }^{71}$ O. Kühn, V. Sundström, and T. Pullerits, Chem. Phys. 275, 15 (2002).

${ }^{72}$ T. Kato and Y. Tanimura, Chem. Phys. Lett. 341, 329 (2001).

${ }^{73}$ S. Woutersen and H. J. Bakker, Phys. Rev. Lett. 83, 2077 (1999).

${ }^{74}$ (a) J. Stenger, D. Madsen, P. Hamm, E. T. J. Nibbering, and T. Elsaesser, Phys. Rev. Lett. 87, 027401 (2001); (b) J. Stenger, D. Madsen, J. Dreyer, P. Hamm, E. T. J. Nibbering, and T. Elsaesser, Chem. Phys. Lett. 354, 256 (2002).

${ }^{75}$ S. Yeremenko, M. Pschenichnikov, and D. Wiersma, Chem. Phys. Lett. 369, 107 (2003)

${ }^{76}$ O. Kühn, J. Phys. Chem. A 106, 7671 (2002).

${ }^{77}$ C. C. Marston and G. G. Balint-Kurti, J. Chem. Phys. 91, 3571 (1989). 